\title{
Analysis of Piezoelectric Energy Harvesting Interface Circuit Applied to Automobile Engine Vibration
}

\author{
Zhaona LU'1,a , Junlong Wang, \\ ${ }^{1}$ School of Automotive Engineering, Nantong Institute of Technology, Nantong jiangsu China
}

\begin{abstract}
In order to realize the continuous power supply for the vibration fault monitoring system of automobile engine, aiming at the low efficiency and instability of the existing piezoelectric full bridge rectifier energy collection circuit, this paper proposes a circuit scheme based on synchronous charge extraction. The scheme can provide circuit collection efficiency, and analyze the power of the circuit by impedance analysis. Finally, the experiment shows that the theoretical analysis is consistent with the experimental results. And the synchronous electrical charge extraction circuit can harvest power up to $1.3 \mathrm{~mW}$ under low frequency conditions, which is higher than $0.5 \mathrm{~mW}$ collected by the full-bridge rectifier circuit under the same conditions. The harvested energy meets the power requirements of automotive sensors and microcontrollers.
\end{abstract}

\section{Preface}

Automobile engine is the only power source of traditional fuel vehicle, whose reliability and safety performance are related to the economy, safety and power of the vehicle, so improving the reliability of the engine can guarantee the safe use of the vehicle. The general mechanical failure of the engine can be reflected by its vibration frequency and amplitude, so the running state of the engine can be understood by monitoring the vibration characteristics of the engine with electronic equipment. However, for electronic devices assembled in mechanical parts, it is difficult to use traditional chemical batteries to achieve continuous power supply due to their limited capacity and difficult disassembly and assembly. Therefore, other forms of energy supply must be sought [1-5].

There is a lot of vibration energy in the vehicle due to the change of speed and direction of the vehicle in the bumpy traffic, and the internal excitation of the engine, gearbox and chassis. The vibration caused by the engine occupies the majority of the vibration of the car ${ }^{[6]}$. Through the transducer, the vibration energy generated by the engine is fed to the wireless sensor to realize the continuous power supply of electronic equipment.

Broadly speaking, any electromechanical transducer can be used to capture the vibration energy produced by an automobile engine. At present, three conversion mechanisms, respectively known as piezoelectric conversion, magneto-electric conversion and electrostatic conversion, have been widely studied. Piezoelectric ceramics are the easiest of the three conversion mechanisms, so they are suitable for large-scale application in capturing the vibration energy generated by automobile engines. In addition,mitcheson et al. analyzed the performance limits of the three conversion mechanisms and found that the performance of piezoelectric materials was better than the other two power generation methods in terms of low frequency ${ }^{[7-8]}$.

In the piezoelectric vibration energy harvesting interface circuit, the Standard Energy Harvesting circuit(SEH), which is made up of rectifier bridge and filter capacitor, is proposed by G.K.Ottman ${ }^{[9]}$, but its harvesting efficiency is poor. Synchronous Switch Harvesting on Inductor (SSHI) can alter the output voltage direction and increase the output voltage of synchronous piezoelectric voltage by means of series R-L-CP circuit oscillation, and improve the efficiency of piezoelectric energy harvesting. According to their positions, SSHI can be classified into series and parallel models, including s-synchronized Switch Harvesting on Inductor and p-synchronized Switch Harvesting on Inductor. The interface circuit of the piezoelectric energy has a defect that when the load resistance change, their output changes accordingly while can not keep the maximum power output.therefore, in practical application Maximum Power Point Tracking (MPPT), This greatly increases the complexity of circuit applications. Synchronous Electric Charge Extraction [11] ( SECE) adopts active switch and synchronous inductance, and the output voltage of the piezoelectric chip rapidly drops to 0 at the synchronous time, keeping the vibration energy extracted same each time. Thus the output power is independent of the load, and is consistent and stable at the maximum power extraction point. Therefore, the application scenarios are greatly increased, and the energy collection efficiency is high.

aemail: luzhn@ntit.edu.cn, bemail:wangj1152213@163.com, 


\section{Vibration response of automobile engine}

The engine is a very complex mechanical system. In order to simplify the vibration of the engine, we will focus on the vibration of the engine in the Z-axis direction. In this paper, SAIC volkswagen's EA211 is adopted as the research object to record vibration signals at different speeds.

Fig. 1(a) shows the acceleration amplitude diagram of the engine in the $\mathrm{Z}$-axis direction when the vehicle is running at $1000 \mathrm{r} / \mathrm{min}$. From the figure we can see that the amplitude is roughly like a sinusoidal waveform of a particular frequency, with some burrs superimposed. For this reason, the fourier transform of Figure1(a) is used to analyze the frequency domain characteristics of vibration signals. The results in Fig1(b) indicate that the engine vibration has resonance frequency. It can be simulated as a sinusoidal signal vibration, and its resonance frequency is in the low frequency range (less than $100 \mathrm{~Hz}$ ) resonance frequency. Therefore, the piezoelectric energy harvesting circuit is designed to work in the $Z$ direction,to operate at low frequency and extract energy.

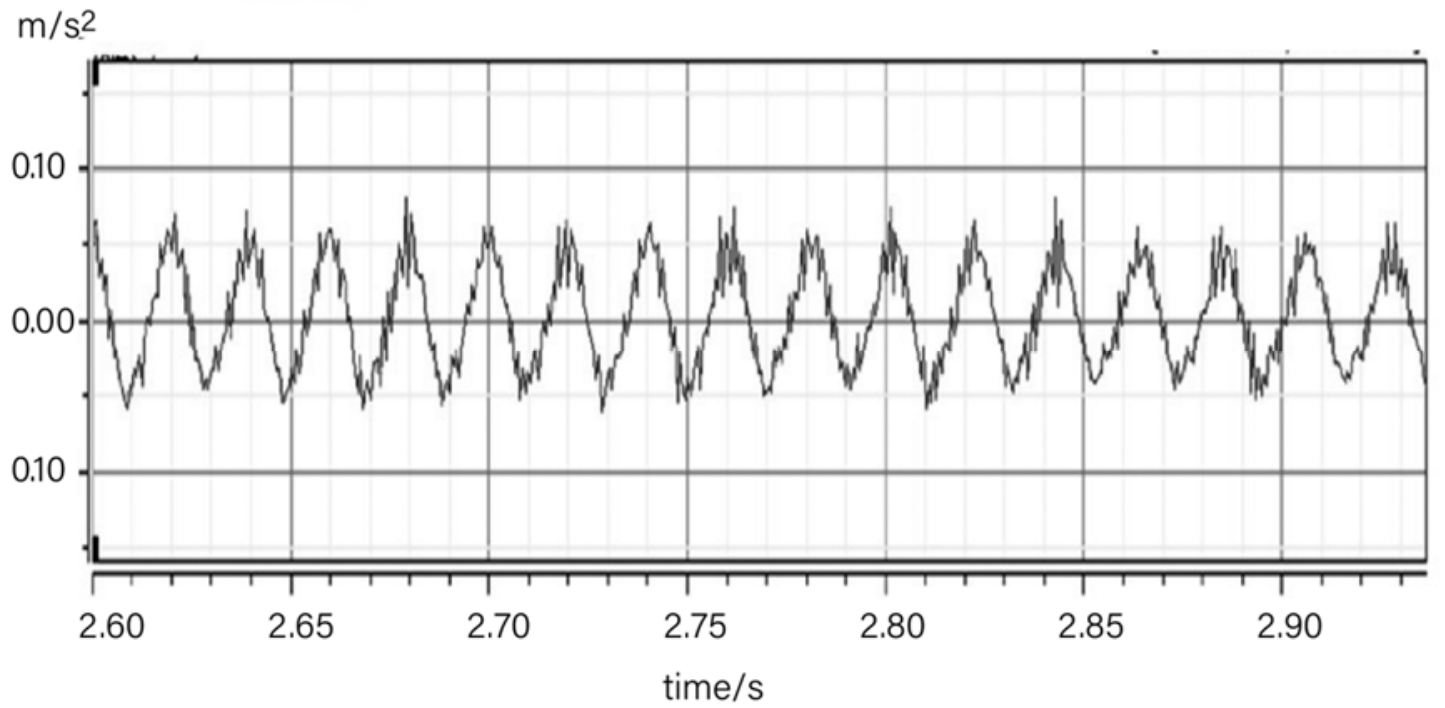

(a)

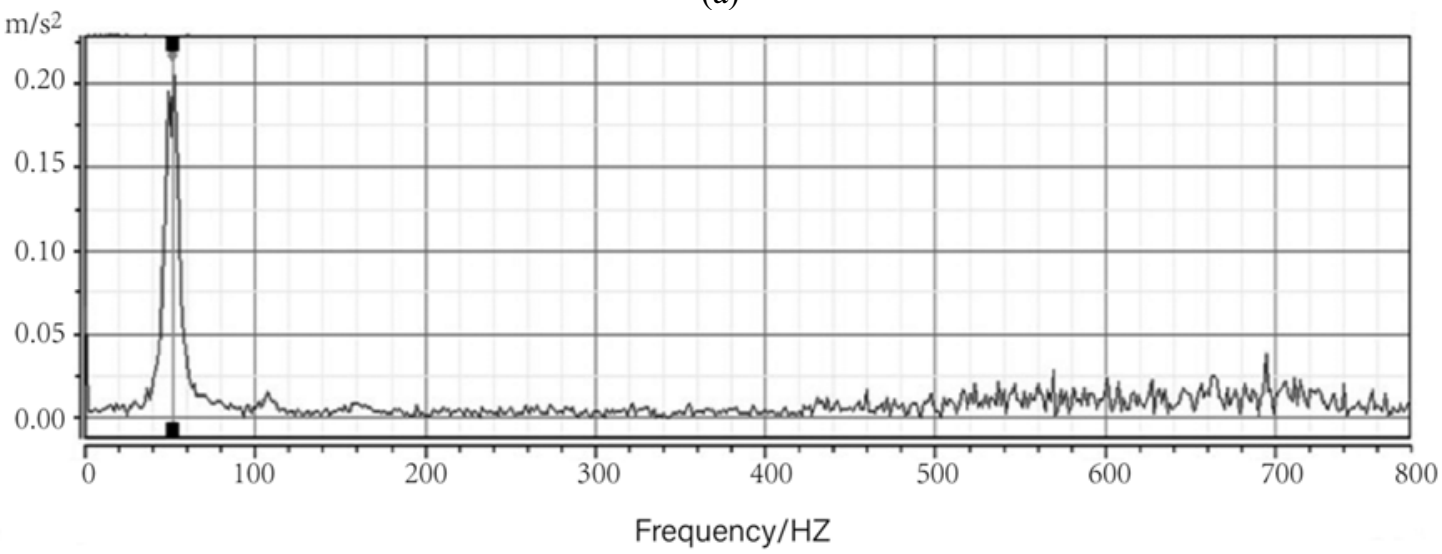

(b)

Fig. 1 Amplitude of Z-axis acceleration of vehicle at 1000r/min (A) Time domain; (B) Frequency domain

\section{Piezoelectric energy harvesting circuit}

From the perspective of application, a complete piezoelectric energy harvesting system can be divided into five parts and two different energy fields. This is shown in Figure 2. The engine of a car produces mechanical vibration. The whole engine can be regarded as mechanical energy. It is the source of the whole energy. Piezoelectric transducer is a key component in the PEH collection system. Through piezoelectric effect, the mechanical energy of the engine is converted into electric energy, so it contains both mechanical energy and electric energy. The main work of the interface circuit is to realize the conversion of $\mathrm{AC}$ to $\mathrm{DC}$ the conversion of $\mathrm{AC}$ generated by piezoelectriv materials to DC used by automobile sensors. The energy storage unit stores the converted electrical energy for use by DC load such as car sensors. The interface circuit, the energy storage, and the DC load are all in the power domain.

The easiest way to convert AC to $\mathrm{DC}$ is to use full bridge rectifier. This AC-DC conversion mechanism only requires a rectifier bridge composed of four diodes. However, due to its extremely low energy collection efficiency, it can not provide continuous and stable energy for DC load. At the same time, the AC electricity generated by piezoelectric materials is different from the 
traditional AC electricity, which has the characteristics of low frequency, high voltage and low current. In order to improve the efficiency of energy collection, a series of piezoelectric energy interface circuits are designed.
This time in the automotive engine energy collection, we use the Synchronous Electric Charge Extraction circuit (SECE).

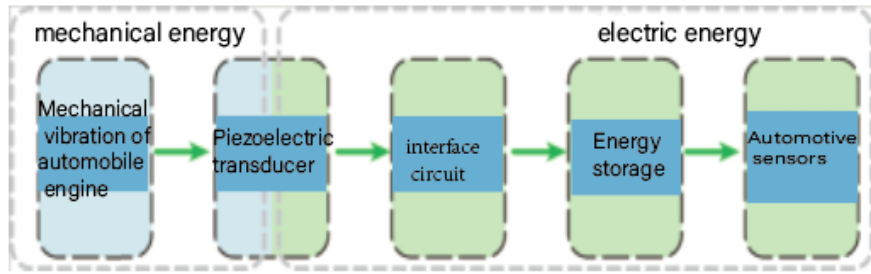

Fig. 2 Energy flow in a piezoelectric energy harvesting system

The SECE circuit, as shown in Figure 3, consists of a rectifying bridge, a switch $\mathrm{S}$, an inductor $\mathrm{Li}$, a continuation diode $\mathrm{D}$, and a filtering current $\mathrm{Cr}$. Because

of the phase difference of current and voltage in the conventional AC-DC rectifier bridge,

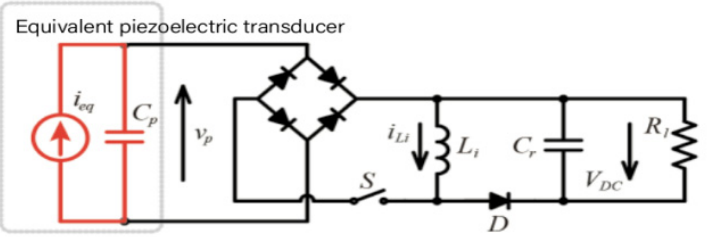

(a)



(b)

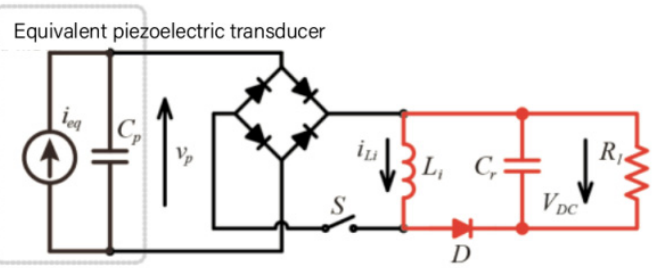

(c)

Fig. 3 Energy flow of synchronous charge extraction circuit at different stages (a) Open circuit state; (b) Switch closed state; (c) Continuing flow stage

The energy collection efficiency of the rectifier bridge reaches low.Is caused by the low energy collection efficiency of the rectifier bridge. To improve the energy collection efficiency, synchronous charge extraction circuit introduces synchronous switch $\mathrm{S}$, so that the current IEQ and voltage VP have no phase difference, and the positive half period and the second half period of engine vibration are both output positive work, which finally improves the efficiency of vibration energy collection.

In a complete vibration cycle, the work of the SECE synchronous charge extraction circuit can be divided into three stages. In Fig. 3 (a), in the positive half period of vibration, the synchronous switch $\mathrm{S}$ is open. With the continuous accumulation of charges at both ends of the capacitor $\mathrm{Cp}$, the voltage of the piezoelectric plate continues to rise. At this point, the synchronous switch $\mathrm{S}$ is closed, and the SECE circuit enters the switch closure state of the second stage, as shown in Figure 3 (b). The capacitor $\mathrm{Cp}$ and inductance $\mathrm{Li}$ form the $\mathrm{L}-\mathrm{C}$ oscillator circuit because of the closing of the switch $\mathrm{S}$. The accumulated mechanical energy on the capacitor $\mathrm{Cp}$ is quickly transferred to the inductor for storage, as shown in a partial enlargement of Figure 4. Because the energy transfer time is very short compared to the whole low-frequency vibration time, it can be regarded as instantaneous energy transfer. Therefore, VP voltage drops rapidly to 0 , that is, at $\mathrm{T} / 2$ in Fig. 4 . Then the synchronous switch $\mathrm{S}$ is disconnected, and the synchronous charge SECE circuit enters the third stage, the current continuation stage. The accumulated energy in the inductor $\mathrm{Li}$ is transferred to the $\mathrm{DC}$ load $\mathrm{RL}$ through the continuous-current diode for subsequent DC loads. In the second half of the vibration period, previous three stages will also occur so there are two energy extractions in a complete vibration period.

By introducing the same switch, the piezoelectric current IEQ and piezoelectric voltage VP always maintain the same phase during the energy conversion process, so the energy collection efficiency can be greatly improved. 




Fig. 4 Current and voltage waveform of synchronous charge extraction circuit

\section{Power analysis of interface circuit}

In the process of piezoelectric energy harvesting, the conversion of mechanical energy and electrical energy exists. The analysis of piezoelectric energy collection power is different from the traditional current and voltage analysis method. Since there is energy loss in the process of energy transfer, we need to take the on-voltage drop of the rectifier bridge and the energy loss of the parasitic resistance into consideration, and use the impedance analysis method for the analysis ${ }^{[12]}$.

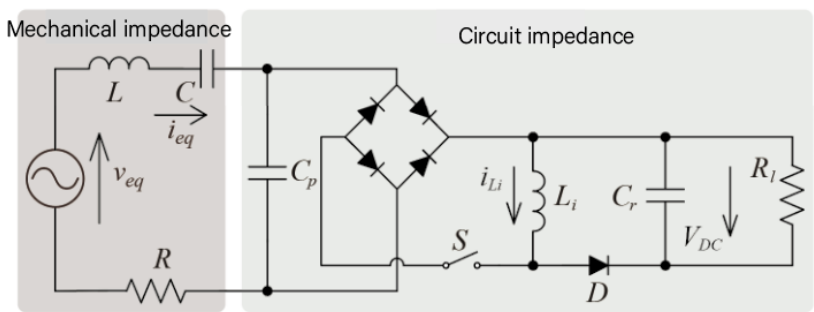

Fig. 5 Synchronous charge extraction circuit impedance

In the impedance analysis method, the electromechanical PEH system is divided into two parts, as shown in Fig. 5. The piezoelectric plate is equivalent to the series structure of voltage source VEQ, inductor L, resistor $\mathrm{R}$ and capacitor $\mathrm{C}$. Their values are:

$$
\begin{gathered}
L=\frac{M}{\alpha_{e}^{2}} \\
C=\frac{\alpha_{e}^{2}}{K} \\
R=\frac{D}{\alpha_{e}^{2}}
\end{gathered}
$$

Where $\mathrm{M}$ is the equivalent mass of the piezoelectric material, $\mathrm{K}$ is the elastic coefficient of the piezoelectric mechanical structure, and D is the mechanical damping. So we convert physical quantities in the mechanical domain to physical quantities in the electrical domain. Finally, the electromechanical piezoelectric energy harvesting system will be converted into a pure electric system, which is more conducive to the analysis of the collected power by using pure circuit. Impedance expression is,

$$
\begin{gathered}
X_{L}=\frac{\omega M}{\alpha_{e}^{2}} \\
X_{C}=\frac{K}{\omega \alpha_{e}^{2}}
\end{gathered}
$$

For the circuit part, due to the existence of non-linear components such as rectifier bridge and continuous-current diode $\mathrm{D}$, the power analysis is a little difficult to do. Therefore, according to the principle of energy conservation in the $\mathrm{PEH}$ system, the circuit part is equivalent to the series form of capacitor $\mathrm{Xe}$, resistor $\mathrm{Rh}$ and resistor $\mathrm{Rd}$. Their representation is as follows

$$
\begin{gathered}
X_{E}=-\frac{1}{\omega C_{p}} \\
R_{h}=\frac{4}{\pi} X_{E} \gamma\left(1-\frac{V_{D}}{V_{D C}+V_{D}}\right)\left(1-V_{F}^{\prime}\right)^{2} \\
R_{d}=-\frac{4}{\pi} X_{E}\left[V_{F}^{\prime}+\left(1+\frac{\gamma V_{D C}}{V_{D C}+V_{D}}\right)\left(1-V_{F}^{\prime}\right)\right]
\end{gathered}
$$

Among them

$$
V_{F}^{\prime}=\frac{V_{F}}{V_{o c}}
$$

Represents the ratio of the tube voltage drop Vf of the continuation diode to the open circuit voltage VOC of the piezoelectric material. Therefore, the expression of the collected power of the synchronous charge extraction circuit SECE of the piezoelectric material applied to the vibration of the automobile engine is

$$
P_{h}=\frac{V_{e q}^{2}}{2} \frac{R_{h}}{\left(X_{L}+X_{C}+X_{E}\right)^{2}+\left(R_{h}+R_{d}+R\right)^{2}}
$$

The expressions of XL, XC and XE are shown in Equations (4), (5) and (6).

\section{Experimental analysis}

The experimental device is shown in Fig. 6. We used a 
shaker to simulate an automobile engine in motion, and it could output a low-frequency sinusoidal vibration signal consistent with Fig. 1. One end of the piezoelectric plate is fixed on the base, so that the piezoelectric material can vibrate with the shaker. The fixed end of the piezoelectric material leads out two electrodes and is connected with the synchronous charge extraction circuit SECE to collect the energy of the shaker vibration. Accelerometer sensors are mounted on the base to monitor the acceleration of the entire PEH energy harvesting system.

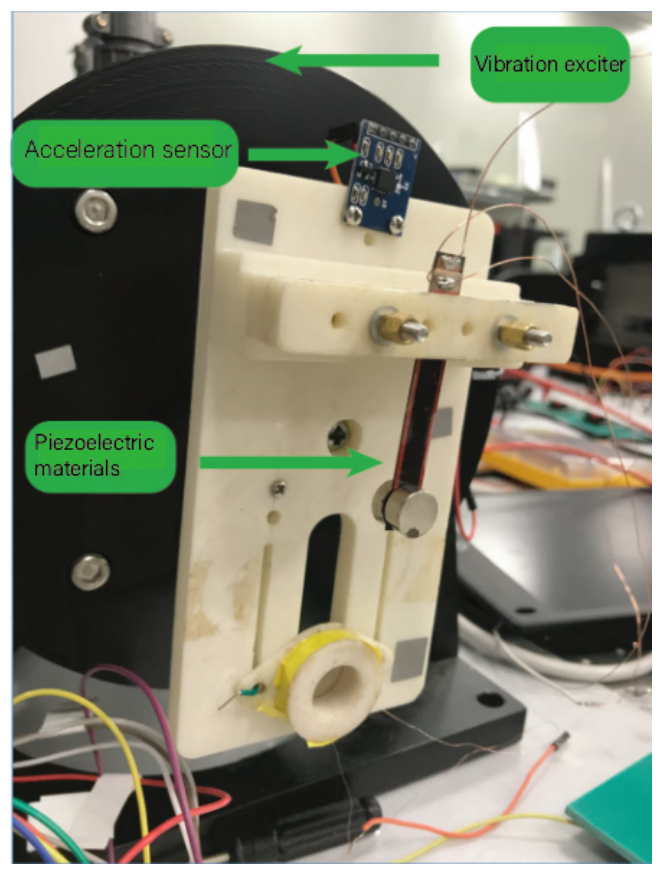

Fig. 6 Experimental apparatus

The experimental waveform of the circuit is shown in Fig. 7 (a). The output voltage Vp of the piezoelectric slice first rises to the peak and then immediately drops to 0 . The waveform of the synchronous charge circuit is consistent with that in Fig. 4, which verifies that the circuit work normally. In Fig. 7 (b), the actual collected power of the synchronous charge circuit is $1.3 \mathrm{~mW}$, while the collected power of the interface circuit only using the rectifier bridge is only $0.5 \mathrm{~mW}$. Therefore, the synchronous charge extraction circuit can greatly improve the power of energy collection. The experimental collected power is consistent with the theoretical collected power, which proves the feasibility of impedance analysis method.

\section{Conclusion}

In this paper, we study the piezoelectric energy harvesting circuit applied to automotive engine vibration. It is found that the vibration of automobile engine has a resonance frequency, which can be simulated as a low-frequency sinusoidal signal vibration, and the working environment of the piezoelectric energy harvesting circuit is determined. For this reason, we choose the synchronous circuit extraction circuit as the interface circuit for energy collection and use impedance analysis method to analyze the electromechanical combined PEH collection system. The final experimental results verify the theoretical analysis. The piezoelectric synchronous charge extraction circuit has a collection power of $1.3 \mathrm{~mW}$, which is sufficient for the MSP430 with $0.66 \mathrm{~mW}$ operating power and $0.33 \mathrm{uW}$ low power mode. Therefore, it is fully capable of supplying power to sensor components or SCM.

\section{Acknowledgents:}

This article is one of the phase results of 《Young and Middle-aged Key Teachers of Nantong Institute of Technology (ZQNGG205) and 《Nantong Key Laboratory of New Energy Vehicle Digital Development and Performance Testing Technology》 (CP12017003).

\section{References:}

1. Mateu L, Echeto F M. Review of energy harvesting techniques and applications for microelectronics $[\mathrm{J}]$ Proceedings of SPIE - The International Society for Optical Engineering, 2005, 5837:359-373.

2. Thomas J P, Qidwai M A, Kellogg J C . Energy scavenging for small-scale unmanned systems[J]. Journal of Power Sources, 2006, 159(2):1494-1509.

3. Penella $\mathrm{M} \mathrm{T}$, Gasulla M .2007 IEEE Instrumentation \& Measurement Technology Conference IMTC 2007 - A Review of Commercial Energy Harvesters for Autonomous Sensors[J]. 2007:1-5.

4. Gilbert J M, Balouchi F . Comparison of energy harvesting systems for wireless sensor networks $[\mathrm{J}]$. International Journal of Automation and Computing, 2008, 5(4):334-347.

5. Jia D , Liu J . Human power-based energy harvesting strategies for mobile electronic devices[J]. Frontiers in Energy, 2009, 3(1):27-46.

6. TIAN Ying, LIU Jun, LUO Guobiao. Experimental Research and Analysis of Vehicle Engine Vibration System [J]. Journal of Foshan University of Science and Technology: Natural Science, 2018, 36(2): 42-47.

7. Chen Yongjun, 2, Wenquan, et al. A MEMS piezoelectric energy collector based on fault monitoring $[\mathrm{J}]$. Piezoelectrics \& Acousto-optics, 2017, 39(3):383-386.

8. Hamza, R. and Robin, L. 2009, MEMS energy harvesting devices, technologies and markets, Technical report, Yole Development.

9. G. K. Ottman, H. F. Hofmann, A. C. Bhatt, and G. A. Lesieutre, "Adaptive piezoelectric energy harvesting circuit for wireless remote power supply," IEEE Trans. Power Electron., vol. 17, no. 5, pp. 669-676, Sep. 2002.

10. Shu Y C, Lien I C, Wu W J . An improved analysis of the SSHI interface in piezoelectric energy harvesting $[\mathrm{J}]$. Smart Materials and Structures, 2007, 16(6):2253-2264. 
11. Lefeuvre, E., "Piezoelectric energy harvesting device optimization by synchronous electric charge extraction," J. Intell. Mater. Syst. Struct. 16(10), 865-876 (2005).

12. Zhao, Y., Zhou, C., and Liang, J., "Implementation of synchronized triple bias-flip interface circuit towards higher piezoelectric energy harvesting capability," in [Proceedings of the 26th International Conference on Adaptive Structures and Technologies], ICAST (2015). 